\title{
LETTER
}

\section{Criticizing reporting standards fails to improve quality in animal research}

\author{
Gordon P Otto ${ }^{1,2}$ and Ralf A Claus ${ }^{1,2^{*}}$ \\ See related research by Barra and Joffe, http://ccforum.com/content/18/1/R15
}

We agree with Bara and Joffe that there is a need for improvement of reporting quality in animal research (AR) [1]. Presenting details on both methods used and potential cofounders during AR is not only important to reproduce results, and to ensure animal welfare (AW) and public support, it is a duty when animals are compromised, stressed or sacrificed to understand diseases or to identify treatment targets.

The article addresses important issues, unfortunately without a solution and ignoring personal responsibility. As mentioned, the word count within manuscripts is strictly limited. In Germany and most European countries, the approval procedure for AR includes applications ( $>6,000$ words) covering all analysed readouts: anaesthesia, pain control, euthanasia methods, termination criteria, statistical planning, funding, discussion of reduction, refinement and replacement, and a systematic review. Nevertheless, how should these long method descriptions be included in manuscripts of 3,500 words?

Really what are required are special conferences and articles focusing on methods in AR, a uniform summarising data sheet as supplementary material, and the presentation of the registration number given by the AW committee including a recheck by the committees to ensure that AW was considered. Finally, there is a need for commitment among scientists to standardise experiments to allow collaborative exchange of data, body fluids and tissues to privilege synergetic benefits; to improve the informative value of an approach by stratification of animals [2]; and to also present negative results to avoid double testing. These changes will lead to increased quality in reporting, realisation of reduction, refinement and replacement, and public perception.

\section{Authors' response \\ Meredith Bara and Ari R Joffe}

We agree with Otto and Claus that it is our moral duty to take sentient animals' interests into consideration when performing AR that can cause suffering and early death. However, Otto and Claus believe we have not discussed 'a solution', and suggest that 'a uniform summarising data sheet as supplementary material and the presentation of the registration number given by the AW committee' would be steps forward. Although these would be welcome steps, they are not sufficient.

We do not believe that the approval procedure for $\mathrm{AR}$, including the longer submissions to the AW committee, can account for the poor ethical reporting we identified. For example: euthanasia methods in our

\footnotetext{
* Correspondence: ralf.claus@med.uni-jena.de

${ }^{1}$ Center for Sepsis Control and Care, Jena University Hospital, Erlanger Allee 101, 07747 Jena, Germany

${ }^{2}$ Clinic for Anesthesiology and Intensive Care, Jena University Hospital, Erlanger Allee 101, 07747 Jena, Germany
}

review were often reported, yet not the appropriate method [1]; few systematic reviews of AR are published, most of low quality, and it is unlikely that the highquality systematic reviews are presented to the AW committee yet never published [3]; reporting statistical planning and sample size calculations do not take significant space in manuscripts yet markedly improve internal validity, making nonreporting hard to explain; and there are problems with the AW committee approval process, including conflicts of interest, lack of strong public representation, and a culture of acceptance that AR is necessary in almost any circumstance $[4,5]$. Indeed, the poor reporting we identified may be a sign of poor performance of AW committees in general. Our 'solution' is thus for journals and reviewers to mandate adequate AR justification and reporting in manuscripts $[1,6]$. 


\section{Abbreviations}

AR: Animal research; AW: Animal welfare.

\section{Competing interests}

The authors declare that they have no competing interests.

\section{Published: 31 Mar 2014}

\section{References}

1. Bara M, Joffe AR: The ethical dimension in published animal research in critical care: the public face of science. Crit Care 2014, 18:R15.

2. Osuchowski MF, Connett J, Welch K, Granger J, Remick DG: Stratification is the key: inflammatory biomarkers accurately direct immunomodulatory therapy in experimental sepsis. Crit Care Med 2009, 37:1567-1573.

3. Lamontagne F, Briel M, Duffett M, Fox-Robichaud A, Cook DJ, Guyatt G, Lesur O, Meade MO: Systematic review of reviews including animal studies addressing therapeutic interventions for sepsis. Crit Care Med 2010, 38:2401-2408.

4. Russell D: Why animal ethics committees don't work. Between Species 2012, 15:127-142.

5. Schuppli CA, Fraser D: Factors influencing the effectiveness of research ethics committees. J Med Ethics 2007, 33:294-301.

6. Osborne NJ, Payne D, Newman ML: Journal editorial policies, animal welfare, and the 3Rs. Am J Bioeth 2009, 9:55-59.

$10.1186 / \mathrm{cc} 13804$

Cite this article as: Otto and Claus: Criticizing reporting standards fails to improve quality in animal research. Critical Care 2014, 18:421 